\title{
BMJ Global Health Global, regional and national burden of emergency medical diseases using specific emergency disease indicators: analysis of the 2015 Global Burden of Disease Study
}

\author{
Junaid Razzak, ${ }^{1}$ Mohammad Farooq Usmani, ${ }^{2}$ Zulfiqar A Bhutta ${ }^{3}$
}

\begin{abstract}
To cite: Razzak J, Usmani MF, Bhutta ZA. Global, regional and national burden of emergency medical diseases using specific emergency disease indicators: analysis of the 2015 Global Burden of Disease Study. BMJ Glob Health 2019;4:e000733. doi:10.1136/ bmjgh-2018-000733
\end{abstract}

Handling editor Seye Abimbola

- Additional material is published online only. To view please visit the journal online (http://dx.doi.org/10.1136/ bmjgh-2018-000733).

Received 12 February 2018 Revised 1 October 2018 Accepted 6 0ctober 2018

Check for updates

(c) Author(s) (or their employer(s)) 2019. Re-use permitted under CC BY-NC. No commercial re-use. See rights and permissions. Published by BMJ.

${ }^{1}$ Emergency Medicine, Johns Hopkins University, Baltimore, Maryland, USA

${ }^{2}$ Johns Hopkins University School of Medicine, Baltimore, Maryland, USA

${ }^{3}$ The Centre for Global Child Health, Hospital for Sick Children, Toronto, Ontario, Canada

Correspondence to Dr Junaid Razzak; junaid.razzak@jhu.edu

\section{ABSTRACT}

Objective There are currently no metrics for measuring population-level burden of emergency medical diseases (EMDs). This study presents an analysis of the burden of EMDs using two metrics: the emergency disease mortality rate (EDMR) and the emergency disease burden (EDB) per 1000 population at the national, regional and global levels. Methods We used the 1990 and 2015 Global Burden of Disease Study for morbidity and mortality data on 249 medical conditions in 195 countries. Thirty-one diseases were classified as 'emergency medical diseases' based on earlier published work. We developed two indicators, one focused on mortality (EDMR) and the other on burden (EDB). We compared the EDMR and EDB across countries, regions and income groups and compared these metrics from 1990 to 2015 .

Results In 2015, globally, there were 28.3 million deaths due to EMDs. EMDs contributed to $50.7 \%$ of mortality and $41.5 \%$ of all burden of diseases. The EDB in low-income countries is 4.4 times that of high-income countries. The EDB in the African region is 273 disability-adjusted life years (DALYs) per 1000 compared with 100 DALYs per 1000 in the European region. There has been a $6 \%$ increase in overall mortality due to EMDs from 1990 to 2015 . Globally, injuries (22\%), ischaemic heart disease $(17 \%)$, lower respiratory infections $(11 \%)$ and haemorrhagic strokes (7\%) made up about $60 \%$ of EMDs in 2015.

Conclusion Globally, EMDs contributed to more than half of all years of life lost. There is a significant disparity between the EDMR and EDB between regions and socioeconomic groups at the global level.

\section{INTRODUCTION}

The emergency medical system is responsible for providing critical, time-sensitive medical care to improve the chances of disability-free survival. The emergency medical system consists of a unique set of steps focusing on the care of patients and making decisions which are initiated at home or the scene of

\section{Key questions}

What is already known?

- Care of patients with sudden-onset emergency medical diseases is a key function of any health system.

- The burden of diseases attributable to these emergency medical diseases is unknown for some high-income countries.

What are the new findings?

- We present two indicators for uniform measurement of the burden of emergency care at the national, regional and global level.

- We found that the burden of emergency medical diseases is two to three times as high in low-income countries of Africa and Asia as in high-income countries.

What do the new findings imply?

- This manuscript presents, for the first time, a summary statistic capturing the global, regional and national figures for emergency medical diseases and could significantly impact future policies on emergency health system especially in countries with high burden of such diseases.

illness/injury, continued during transport to the appropriate (often not the nearest) health facility, and provision of life-saving emergency care at the hospital within seconds, minutes and hours of the onset of symptoms. Globally, there is an increasing focus on strengthening emergency medical systems. ${ }^{1-3}$ This focus is often driven by individual diseases such as trauma, maternal mortality and cardiac arrests, or due to concern for large-scale emergencies and disasters. The underlying system of response to emergency medical diseases (EMDs) remains the same, and hence investments in emergency care are likely to benefit a large number of diseases. ${ }^{24}$ We define EMDs as those where interventions within minutes 
to hours are required to improve health outcomes. There is limited knowledge regarding the public health burden of EMDs, which can be addressed through strengthening of emergency medical systems.

There is currently no universally accepted indicator for the burden of EMDs at the population level. The emergency care literature describes emergency care services largely either through measures of service utilisation or through measures of system capacity. Examples of the service utilisation measures include the number of emergency department visits and the number of ambulance transports, while measures of system capacity are often presented as the number of ambulances or the number of emergency department beds. ${ }^{245}$ The population-based burden of EMDs at country and regional levels is unknown. One study reported aggregate data from 2010 Global Burden of Disease (GBD) Study to describe the burden of emergency care at a global level in the context of healthcare utilisation. However, the subanalysis of individual countries was focused on 40 countries mostly from high-income groups and did not include countries from low-income and middle-income group. ${ }^{1}$

The purpose of this study is to describe the burden of EMDs at the national, regional and global level using two novel indicators of the emergency care burden at the population level. The first indicator describes the mortality rates due to EMDs and is called emergency disease mortality rate (EDMR). The second indicator, emergency disease burden (EDB), describes the disability-adjusted life years (DALYs) and years of life lost (YLLs) due to EMDs. The two new indicators are based on existing metrics that are used by the GBD Study. EDMR is based on deaths caused by emergency condition, and EDB is based on DALYs and YLLs. The study proposes these new metrics to compare the aggregate burden of EMDs across countries and regions and thus identify the need to strengthen emergency medical system. We present the analysis of these two indicators based on regions, income levels, gender and age, as well as the changes in these indicators over a 25-year period.

\section{METHODS}

We used the 1990 and 2015 GBD Study, which reports mortality on 249 fatal conditions and morbidity based on 315 non-fatal causes from 195 countries. ${ }^{67}$ Detailed methods for the GBD data collection and estimates have been described elsewhere and include data obtained through a large number of data sources from countries and regions. ${ }^{67}$ These data source include vital registration, verbal autopsy studies, death surveillance and other sources from 1980 to 2015. The GBD Study uses a variety of analytics and data from many different sources to develop reliable estimates of morbidity and mortality across the world, and breaks them down by age, sex, cause, year and geography. The data are published online by the Institute for Health Metrics and Evaluation in the Global Health Data Exchange (GHDx) (Ghdx. healthdata.org/gbd-results-tool). The data were downloaded in January 2017. Briefly, based on the specific disease category, either level 3 or 4 disease group from GBD was used. Level 2 was used for 'Injuries' to include all injuries as a single disease group. The choice of level 3 or 4 disease group was guided by the disease classification from Chang et al. ${ }^{1}$

To identify diseases/conditions to be included in our analysis, we used the earlier work by Chang et $a l^{1}{ }^{1}$ who had carried out a multistep process to divide the 249 medical conditions reported in the GBD into (1) EMDs, (2) non-EMDs but with 'trajectories that can involve acute decompensation' and (3) non-urgent diseases (online supplementary table A). Of the 249 diseases, the authors defined 31 diseases as those fulfilling the definition of EMDs, that is, 'diseases which if not diagnosed and treated within hours to days of onset, often led to serious physical or mental disability or death'. The classification of diseases was carried out through a two-step process. The first step involved classifying all 249 diseases into one of the three categories through a modified Delphi method involving four emergency physicians. This was followed by an external validation process involving 25 physicians involved in the provision of emergency care and represented various regions and income levels. Details of the process are available elsewhere. ${ }^{167}$ To reduce the risk of overestimating the EDB, we decided to include only diseases that were defined as EMDs and did not include conditions that were deemed to be non-emergencies but could become emergency through decompensation.

We used death rates, DALYs and YLLs from 1995 and 2015 from all medical conditions reported in the GBD Study. DALY is a measure of overall disease burden, expressed as the number of years lost due to ill health, disability or early death. ${ }^{8}$ DALY per unit population therefore combines the level of disability and mortality due to each disease. The sum of DALYs across a population indicates the difference between the ideal health of a population and the current health of a population. YLL provides a summary measure of premature mortality. Potential YLL may be defined as the years of potential life lost due to premature deaths. YLL per unit population measures the incidence of lost years of life due to death and gives greater weight to deaths at a younger age and lower weight to deaths at an older age.

Data were downloaded from the GHDx website in absolute numbers, as well as the rate of deaths, YLL and DALYs (per 100000 persons). We combined the rate of death due to EMDs to calculate the EDMR. EDMR was calculated by adding deaths due to EMDs divided by the population and multiplied by 100 . EDB was calculated using DALYs lost due to EMDs. For our analysis, we calculated YLLs or DALYs per 1000 persons by dividing YLLs or DALYs per 100000 by 100 .

For comparison over time, we used the same diseases for the two corresponding periods (1990 and 2015). The only difference between the two years was the absence of reported data on Ebola in 1990. The disease was, 

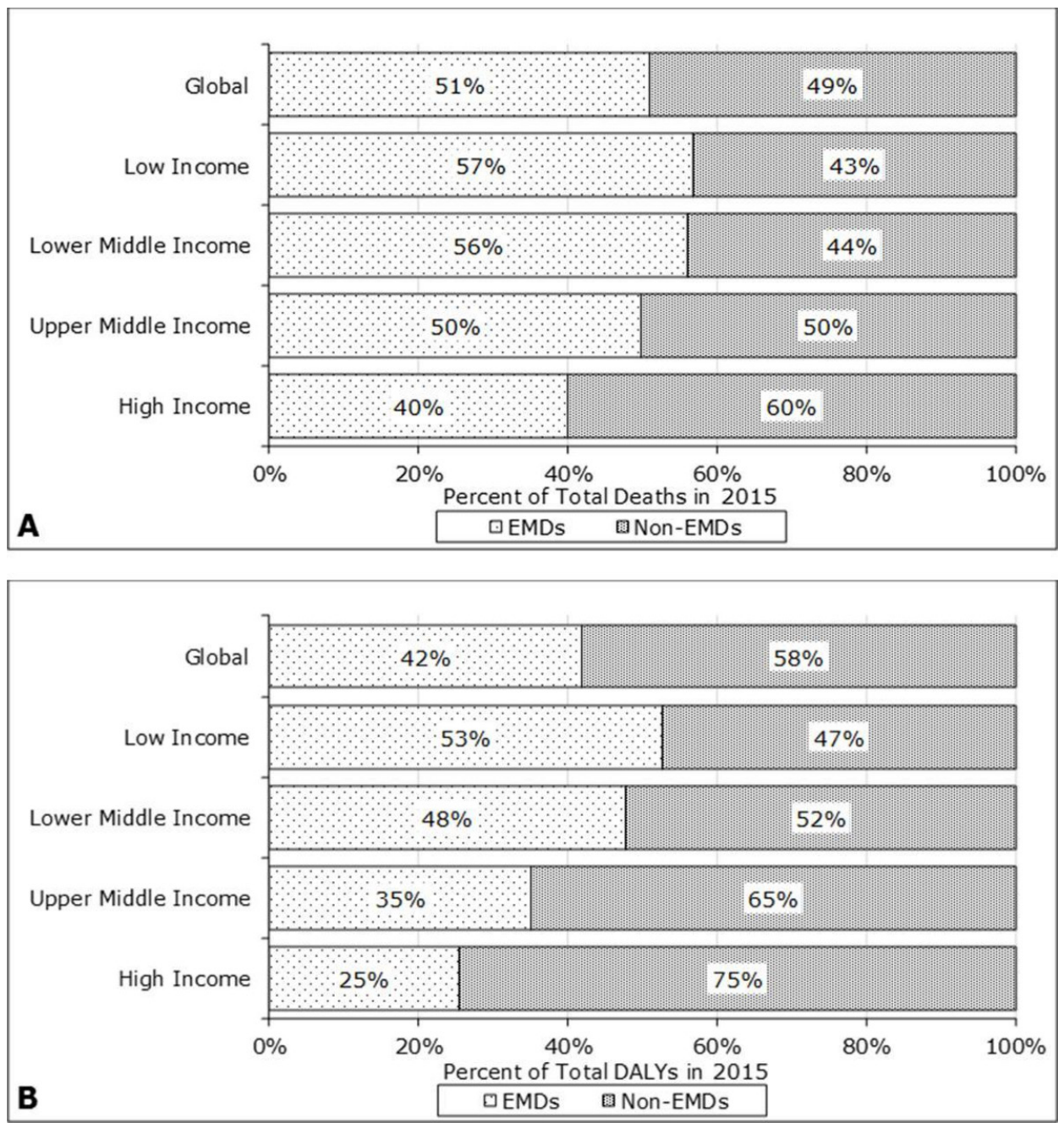

Figure 1 Percent of deaths in 2015 due to emergency and non-emergency medical diseases compared across the different World Bank income groups (A) Emergency medical disease Burden by DALYS in 2015 due to emergency and non-emergency medical diseases compared across the different World Bank income groups (B) EMDs, Emergency medical diseases; NonEMDs, Non-Emergency Medical Diseases. DALYs, disability-adjusted life years.

therefore, excluded from our analysis of the differences between 1990 and 2015. The 95\% CI reported on GHDx was also reported as part of the analysis. The absolute numbers were used to arrive at percentage estimates for disease burden. Finally, the World Bank (WB) income groups (online supplementary table B) and WHO geographical groups (online supplementary table C) were used to compare global data across geographical and income groups. Microsoft Excel and associated graphing functions were used for analysis and charting.

\section{RESULTS}

In 2015 , globally, $50.9 \%$ of mortality (95\% CI $50.2 \%$ to $51.6 \%)$ and $42.0 \%$ of all DALYs (95\% CI $39.0 \%$ to $45.1 \%)$ were caused by EMDs (figure 1). Except in high-income countries, where only $40 \%$ of deaths are caused by EMDs, half or more deaths in upper-middle-income, lower-middle-income and low-income countries are caused by EMDs. Half of all DALYs in low-income and low-middle-income groups are caused by EMDs (52\% and $47 \%$, respectively).

Together low-income and low-middle-income countries, which comprise $49 \%$ of the world's population, contribute to $68 \%$ of DALYs and $70 \%$ of YLLs due to EMDs. Table 1 presents the EDMR and EDB in terms of DALYs and YLLs by WB income groups and WHO regions. The EDB is 4.4 times (in DALYs) and 5.1 (in YLLs) higher in low-income countries compared with high-income countries (282.41 DALYs vs 64.69 DALYs per 1000 and 267.68 YLLs vs 52.46 YLLs per 1000). Overall, at the global level, the EDMR is slightly higher than non-EDMR. The difference is highest again in low-income and lower-middle-income countries, where EDMR is $12 \%$ higher than non-EDMR for both groups.

The EDB remains higher than the non-EDB in low-income countries (4.78 DALYs and 3.64 DALYs per 1000, respectively). Among the WHO's regions, the African region has the highest EDB (275.59 DALYs per 1000), followed by the Eastern Mediterranean region (180.90 
Table 1 EMD burden by mortality rate, DALYs and YLLs per 1000 persons by WB income groups and WHO regions

\begin{tabular}{|c|c|c|c|c|c|c|}
\hline \multirow[b]{2}{*}{ Geographical region } & \multicolumn{2}{|c|}{ Mortality rate } & \multicolumn{2}{|l|}{ DALYs } & \multicolumn{2}{|l|}{ YLLs } \\
\hline & EMDs & Non-EMDs & EMDs & Non-EMDs & EMDs & $\begin{array}{l}\text { Non- } \\
\text { EMDs }\end{array}$ \\
\hline Global & 3.86 & 3.71 & 140.35 & 194.05 & 128.58 & 98.37 \\
\hline \multicolumn{7}{|l|}{ WB income groups } \\
\hline High-income & 3.52 & 5.26 & 64.69 & 190.20 & 52.46 & 81.31 \\
\hline Upper-middle-income & 3.52 & 3.56 & 96.10 & 177.94 & 86.46 & 84.26 \\
\hline Lower-middle-income & 4.09 & 3.21 & 179.95 & 196.63 & 167.17 & 102.37 \\
\hline Low-income & 4.78 & 3.64 & 282.41 & 254.21 & 267.68 & 169.48 \\
\hline \multicolumn{7}{|l|}{ WHO regions } \\
\hline Eastern Mediterranean region & 4.52 & 3.63 & 180.90 & 172.56 & 166.42 & 83.52 \\
\hline African region & 5.09 & 5.04 & 275.59 & 260.29 & 261.82 & 173.27 \\
\hline European region & 2.88 & 3.97 & 101.46 & 199.18 & 87.69 & 91.35 \\
\hline Region of the Americas & 3.99 & 3.45 & 79.40 & 181.60 & 69.65 & 79.77 \\
\hline South-East Asian region & 3.31 & 3.67 & 163.35 & 196.10 & 150.84 & 98.49 \\
\hline Western Pacific region & 3.31 & 3.64 & 83.35 & 168.26 & 74.27 & 77.10 \\
\hline
\end{tabular}

DALYs, disability-adjusted life years; EMDs, emergency medical diseases; WB, World Bank; YLLs, years of life lost; non-EMDs, nonemergency medical diseases.

DALYs per 1000). The difference between emergency disease and non-emergency disease mortality is highest in the Eastern Mediterranean region, followed by the African region and South-East Asian regions (difference: $+56 \%,+22 \%$ and $+15 \%$, respectively) .

Figure 2 presents the changes in the EDMR and the EDB over the 25-year period. Globally there has been a $6 \%$ increase in emergency disease mortality during that period and is most significant in low-income countries
(12\%). The EDB, however, has shown a reduction across all income groups over the past 25 years, with the most significant reductions in upper-middle-income countries.

Figure 3A shows that globally, among WB income groups, four diseases-injuries, ischaemic heart disease (IHD), lower respiratory infections (LRIs) and haemorrhagic strokes-made up about $57 \%$ of all

YLLs in 2015. Neonatal diseases were another big contributor to EMDs and together account for $14 \%$ of

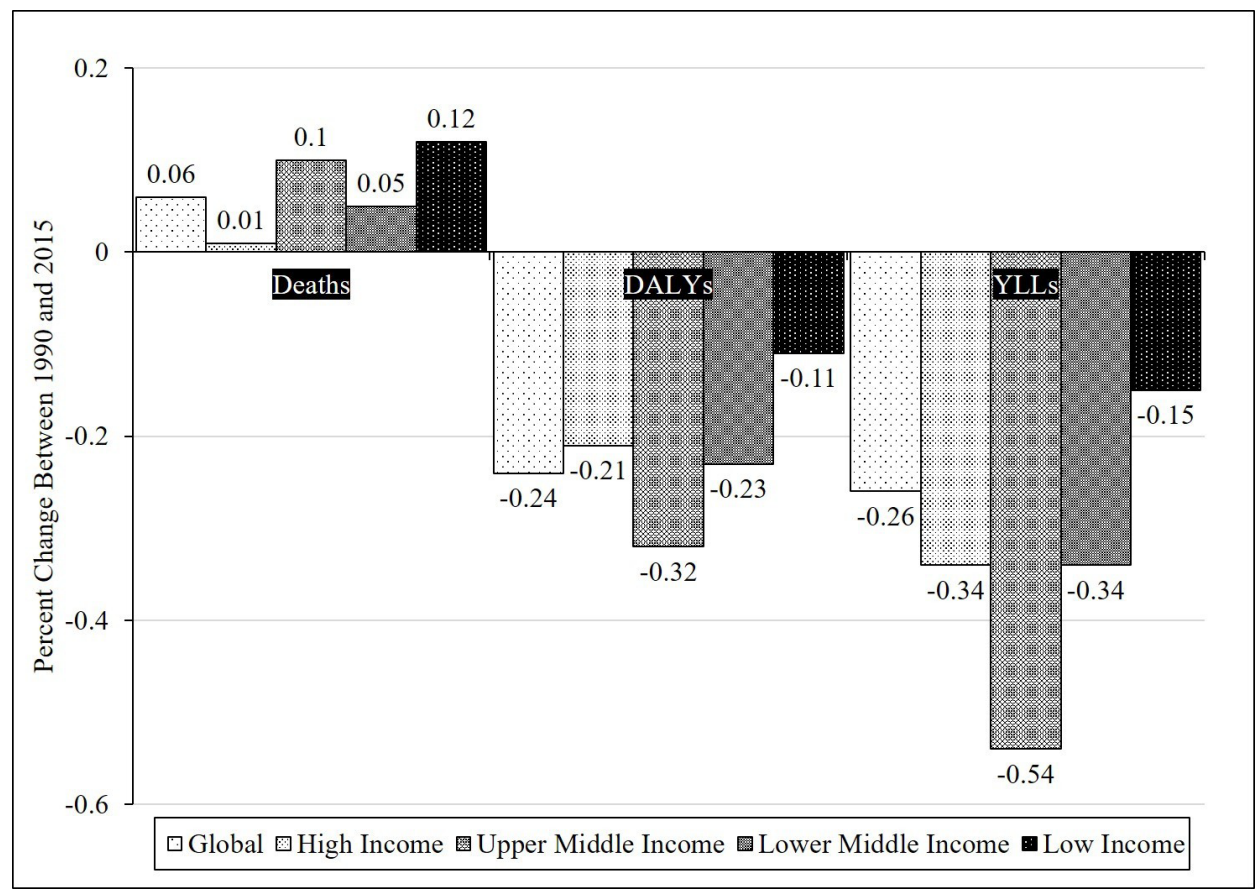

Figure 2 Change in absolute numbers of Deaths, DALYs And YLLs due to Emergency Medical Diseases from 1990 to 2015. DALYs, Disability Adjusted Life Years; YLL, Years of Life Lost. 

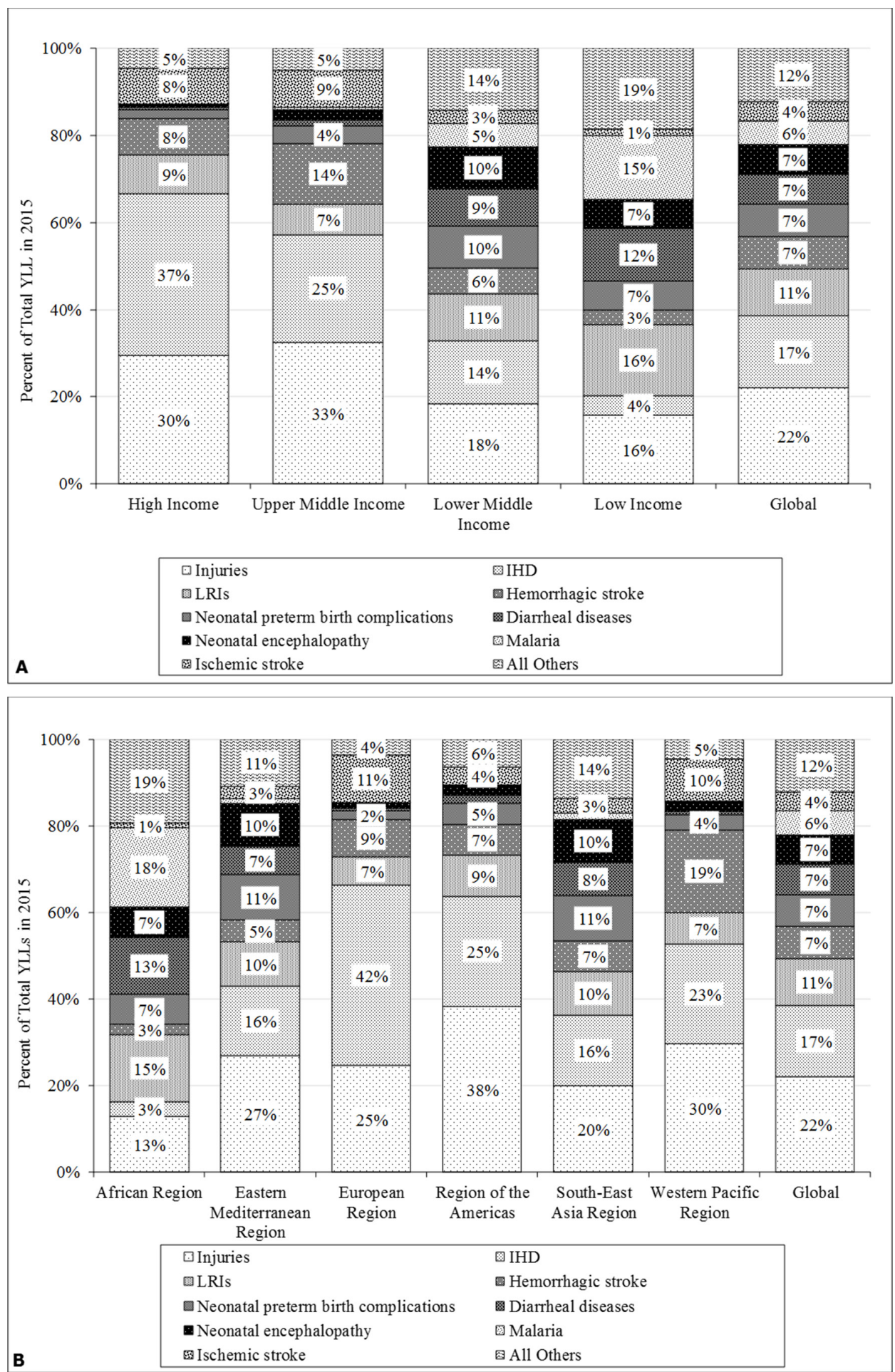

Figure 3 Distribution of Top 10 Emergency Medical Diseases across WB income groups (A) and WHO Geographical Regions (B) WB, World Bank; EMDs, Emergency Medical Diseases; YLL, Years of Life Lost; IHD, Ischemic heart diseases; LRI, Lower Respiratory Infections.

all EMDs. The top 9 EMDs account for about $88 \%$ of all YLLs due to EMDs globally. When the countries are separated by income groups, the trends in high-income and upper-middle-income countries are markedly different from those in low-middle-income and low-income countries. Only four diseases (injuries, IHD, LRIs and haemorrhagic strokes) made up $84 \%$ and $79 \%$ of all EMDs in high-income and upper-middle-income countries, respectively. The four diseases also made up a significant proportion of YLLs due to EMDs in lower-middle-income $(49 \%)$ and low-income $(39 \%)$ countries. However, other diseases such as neonatal diseases, diarrhoeal diseases and malaria become increasingly dominant in these income groups. In low-income regions, neonatal diseases 

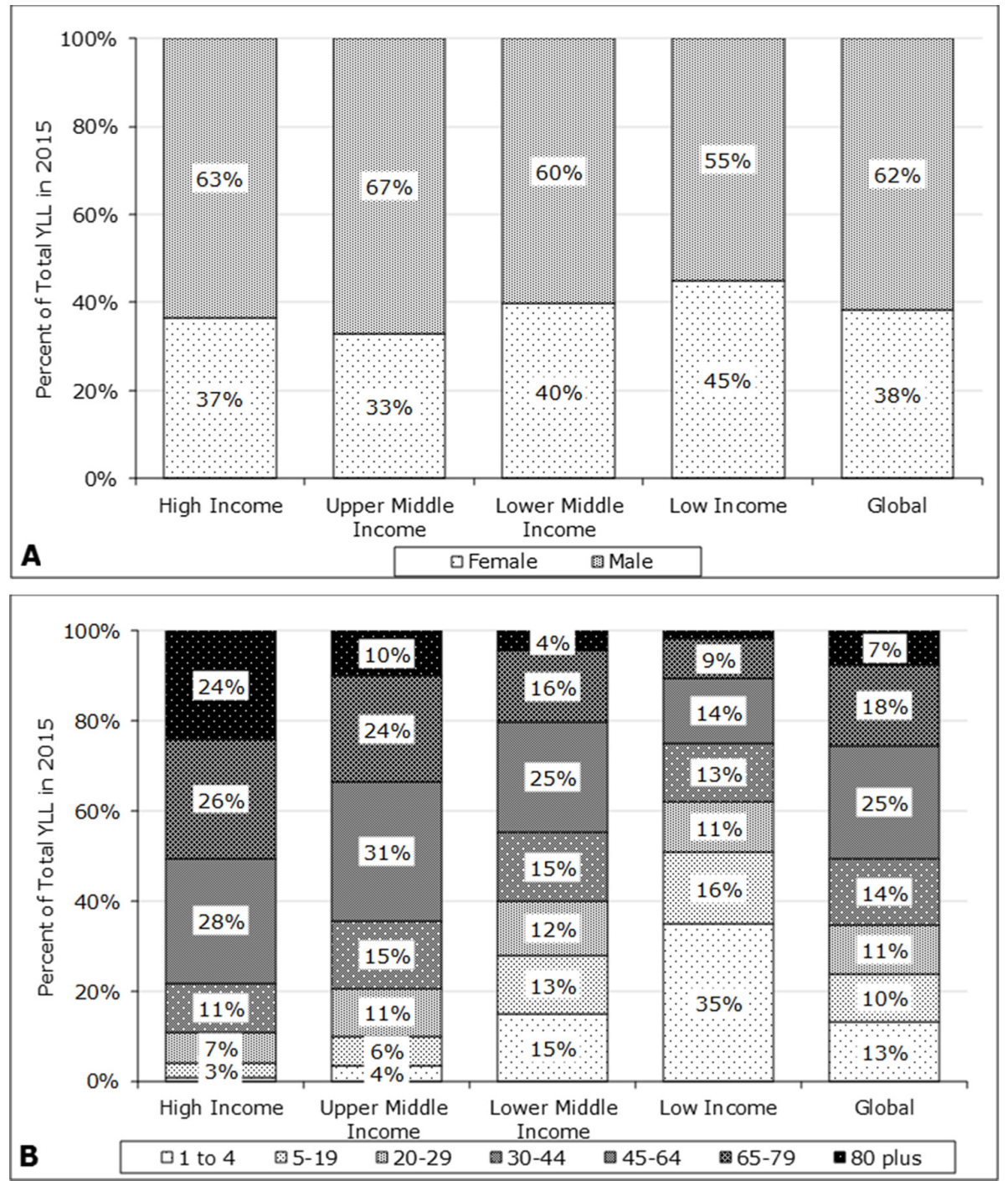

Figure 4 Gender (A) and Age (B) Distribution for total YLL to Emergency Medical Diseases by WB Income Group for the year 2015. EMDs, Emergency Medical Diseases; YLL, years of life lost.

made up a higher percentage of YLLs than IHDs, and haemorrhagic and ischaemic strokes combined (14\% for neonatal diseases vs $8 \%$ for others).

The distribution of EMDs in the different WHO regions varies significantly, as illustrated in figure 3B. In the Western Pacific region, the region of the Americas and the European region, injuries, IHD, LRIs and haemorrhagic strokes made up 79\%, 79\% and $83 \%$ of YLLs, respectively. In contrast, the African region has one of the lowest contributions to EDB from these diseases at just $34 \%$. Unlike the developed regions, neonatal and diarrhoeal diseases made up a significant proportion of the YLLs in the African, South-East Asian and Eastern Mediterranean regions. Finally, malaria is an outlier that seems to cause more morbidity and mortality in the African region than anywhere else in the world.

Figure 4A, B represents EDB for income groups by gender and age. Online supplementary tables 1 and 2A, $\mathrm{B}$ show the comprehensive country-level and regionlevel EDB by DALYs and YLLs by gender and age. EMDs affect males much more than females (DALYs for M:F of 1.43, range: $0.96-2.73)$. Only one country, the Democratic Republic of the Congo, had higher female victims of EMDs than male. Half of all EDBs were seen in people $<45$ years of age. In high-income countries, $17 \%$ of EDB was in patients 44 or younger. By comparison, in low-income countries, $28 \%$ of EDB was in those 44 or younger. The primary difference that accounted for the age distribution of EMDs was driven by children 1-4 years old, followed by children 5-19 years old.

Online supplementary table 1 presents the EDB by DALYs and YLLs compared across gender. The DALYs per 1000 show marked variability across countries. Globally, the countries with the lowest burden of EMDs are Bahrain, Israel and Kuwait (42, 42 and 44 DALYs per 1000, respectively), while Chad, Niger and Mali have the highest EDB (498, 486 and 469 DALYs per 1000, respectively). China, India and the USA, the three most populous countries, have DALYs of 82, 182 and 68 per 1000 s persons and are ranked globally at 64,144 and 47, respectively. 


\section{DISCUSSION}

EMDs contribute to about half of all deaths and almost half of the burden of all diseases globally. This study highlights the disparity of EDB between high-income and low-income countries, as well as the significant disparities across geographical regions. These findings align with earlier studies that investigated the burden in low-income and middle-income countries and determined high patient loads and mortality in emergency care settings. ${ }^{9}$ The data from prior studies were collected from many different sources and based on a sample of the population rather than the whole population. One of the strengths of GBD data, and thus our study, is the population-level analysis. The high percentage of YLLs due to EMDs in lower-middle- and low-income countries makes the case for strengthening the emergency medical care systems in low-income and middle-income countries as outlined in earlier studies. ${ }^{10}$ The exact interventions needed will be dependent on the diseases contributing to the burden; however, the study does highlight the importance of investing in acute care interventions.

While presented as an aggregate based on the acuteness of the illness and the urgency of diagnosis and treatment, the findings are not surprising since key EMDs are already known to be common among low-income and low-middle-income countries. Identifying the disease groups that make large contributions to the healthcare system, however, allows for a health system-based response to the EMDs instead of an approach that focuses on individual diagnoses.

Analysis of the distribution of the EMDs points to variation in the quality of medical care available as well as the health status of the population prior to the development of emergent illness. The leading causes of morbidity due to EMDs were consistent with earlier findings. ${ }^{1-4}$ Injuries, IHD and cardiovascular diseases (CVDs) make up a large percentage of YLLs in high-income and upper-middle-income countries. In lower-middle and low-income countries, however, diseases such as diarrhoea, LRIs, neonatal diseases and malaria make up a significant proportion of YLLs. The disparity in the type of disease contributing to EDB is significant. Interventions needed to prevent multifactorial diseases such as IHD, CVDs and injuries are often costly. ${ }^{11} 12$ They demand more than one type of intervention in order to reduce morbidity and mortality. For example, numerous preventative strategies for IHD are available, but each one can cost between $\$ 1400$ and $\$ 350$ 000 per year of life saved. ${ }^{11}$ Furthermore, one strategy is unlikely to be enough. In addition to smoking cessation, appropriate therapy for hyperlipidaemia and hypertension will also be needed to decrease the risk of IHD. ${ }^{11}$ By contrast, the cost of prevention of the major diseases contributing to high mortality in lower-middle-income and low-income countries is significantly lower. Strategies for prevention of diarrhoea, for example, include water chlorination, ceramic filtration, solar disinfection and flocculation disinfection. The annual cost per person is in the order of tens of dollars per year (\$10-150/year). ${ }^{13} 14$
Therefore, a strategic investment of resources is needed to appropriately tackle the high morbidity in lower-middle-income and low-income countries.

This study also investigated the demographic risk factors for high EDB. Across all income groups, males made up a larger percentage of EDB, likely driven by injuries. Various biopsychosocial explanations can be put forward for the gender differences in diseases burden. Factors such as social support, gender identity, self-esteem, education, exposure to diseases, work responsibilities and many others contribute to the gender differences in morbidity. ${ }^{14-16}$ Our analysis also showed that patients 45 and older contributed most to mortality due to EMDs. In low-income countries, the proportion of younger patients contributing to mortality is higher. Higher mortality for younger patients in low-income countries, due to poor control of modifiable risk factors and lack of access to quality care, has been reported by earlier studies that have analysed the GBD data. ${ }^{17-19}$

Upper-middle-income countries and regions have shown significant improvements in the burden of EMDs. This is likely due to many factors, but probably is related to some extent on the specific attention to emergency care at the population level in the 25 -year period. There are approximately 30 countries in the world where emergency medicine is a specific specialty with formalised residency training, and most of these are clustered in upper-middle-income and high-income countries.

There are several limitations to this analysis. First, the analysis relies on the data obtained from the GBD.

The limitations of the GBD data have been described in detail as part of the initial publication of the GBD results. ${ }^{67}$ These emerged from differences in the sources and quality of data especially those from countries with limited hospital and death registration systems.

Second, categorising diagnoses defined by the GBD study into emergency and non-EMDs is difficult given the fact that many emergency diseases result in chronic sequelae and many chronic diseases can decompensate into emergencies. Despite this limitation, we feel our analysis is valid due to four reasons. First, we have based our categorisation on an earlier published work that used consensus of expert emergency medicine clinicians who represented different clinical settings across the globe. Second, the methodology of the GBD is careful in identifying and recording the 'underlying' cause of death instead of the proximate cause. Thus, diseases that initiate a series of events leading to death are captured in the diagnoses, and in the absence of that underlying disease patients would not have died. ${ }^{20}$ For example, the diagnosis of IHD includes fatal conditions such as cardiac arrest and myocardial infarction, as well as non-fatal yet symptomatic conditions such as ischaemic heart failure and angina. Non-ischaemic causes of heart failure such as hypertension, valvular or rheumatic heart disease are excluded based on available data and through modelled and extrapolated data when actual data are not available. Third, we decided to only focus on 'emergency' 
diseases and excluded those categorised as diseases with a high likelihood of decompensating into a medical emergency. ${ }^{21}{ }^{22}$ This was done to avoid overestimating the burden. Finally, and perhaps most importantly, the purpose of our study is not to capture the 'total' burden of EMDs but rather to present a uniform method and language, with all the known limitations, so that comparisons across time and region can begin. We feel that more refinements will be made as more accurate data are available through sources such as the GBD Study.

The findings of this study have several policy implications. This study presents, perhaps for the first time in peer-reviewed literature, indicators to quantify the burden of EMDs across all countries and regions of the world. Characterising the burden of EMDs with a single metric that is comparable across countries and regions would allow for prioritising emergency care and measuring the effectiveness of investments over time. ${ }^{6} 723$ In addition, it will provide unified language to the governments, international agencies and specialty societies. Prior work that described population-based burden of emergency care has either been limited in scope or did not use standardised indices. Earlier work on the burden of EMDs has been presented for a limited number of countries and primarily focused on the utilisation of resources, covering many high-income countries with few low-income and middle-income countries. ${ }^{1}$

Our findings are not meant to prove attribution of the EDB to the quality of or access to emergency medical care. Higher burden of EMDs perhaps would benefit from public health approaches to preventing emergencies such as road traffic injuries and violence, as well as focusing on emergency care system. Decreasing the burden of EMDs requires interventions at all levels of the healthcare system, from improving preventive care services to increasing patient literacy regarding medical compliance, to optimising delivery of care in emergent situations. Our data do not suggest that improving emergency services alone, during transportation or at the healthcare facility, would result in saving lives and averting the disabilities due to EMDs. Governments and ministries of health need to carefully assess their system's ability to respond to the growing need for emergency care, while specialty societies, academic entities and funding agencies, particularly those with a focus on low-income and lower-middle-income countries, need to increase their attention and investment to further research in this area. Finally, training programmes in healthcare at all levels need to constantly prepare future human resources to respond appropriately to the population needs.

\section{CONCLUSION}

In summary, this study characterised the burden of EMDs across the globe and provided two universal metrics to compare the mortality and the burden of EMDs across countries, regions and income groups. We identified significant disparities. The high mortality due to EMDs makes the case for evidence-based strengthening of the healthcare system, including community-based emergency care, transportation, training communication systems and treatment facilities, as well as focus on upstream modifiable risk factors and downstream acute and rehabilitation services.

Contributors JR conceived this idea, designed the study, participated in the interpretation of data, drafted the manuscript and gave final approval of the manuscript. MFU played a substantial role in the acquisition and analysis of the data, writing of the manuscript, and gave final approval of the manuscript. ZAB was involved in the interpretation of data, reviewed the manuscript and gave final approval of the submission. All agreed to be accountable for all aspects of the work in ensuring that questions related to the accuracy or integrity of any part of the work are appropriately investigated and resolved.

Funding The authors have not declared a specific grant for this research from any funding agency in the public, commercial or not-for-profit sectors.

Competing interests None declared.

Patient consent for publication Not required.

Provenance and peer review Not commissioned; externally peer reviewed.

Data sharing statement No unpublished data from this study are available with anyone.

Open access This is an open access article distributed in accordance with the Creative Commons Attribution Non Commercial (CC BY-NC 4.0) license, which permits others to distribute, remix, adapt, build upon this work non-commercially, and license their derivative works on different terms, provided the original work is properly cited, appropriate credit is given, any changes made indicated, and the use is non-commercial. See: http://creativecommons.org/licenses/by-nc/4.0/.

\section{REFERENCES}

1. Chang CY, Abujaber S, Reynolds TA, et al. Burden of emergency conditions and emergency care usage: new estimates from 40 countries. Emerg Med J 2016;33:794-800.

2. Hirshon JM, Risko N, Calvello EJB, et al. Health systems and services: the role of acute care. Bull World Health Organ 2013;91:386-8.

3. Pines JM, Hilton JA, Weber EJ, et al. International perspectives on emergency department crowding. Acad Emerg Med 2011;18:1358-70.

4. Hsia R, Razzak J, Tsai AC, et al. Placing emergency care on the global agenda. Ann Emerg Med 2010;56:142-9.

5. Jayaprakash N, O'Sullivan R, Bey $\mathrm{T}$, et al. Crowding and delivery of healthcare in emergency departments: the European perspective. West J Emerg Med 2009;10:233-9.

6. Wang $\mathrm{H}$, Naghavi $\mathrm{M}$, Allen $\mathrm{C}$, et al. Global, regional, and national life expectancy, all-cause mortality, and cause-specific mortality for 249 causes of death, 1980-2015: a systematic analysis for the global burden of Disease Study 2015. Lancet 2016;388:1459-544.

7. Kassebaum NJ, Arora M, Barber RM, et al. Global, regional, and national disability-adjusted life-years (DALYs) for 315 diseases and injuries and healthy life expectancy (HALE), 1990-2015: a systematic analysis for the global burden of Disease Study 2015. Lancet 2016;388:1603-58.

8. Murray CJ. Quantifying the burden of disease: the technical basis for disability-adjusted life years. Bull World Health Organ 1994;72:429-45

9. Obermeyer Z, Abujaber S, Makar M, et al. Emergency care in 59 lowand middle-income countries: a systematic review. Bull World Health Organ 2015;93:577-86.

10. Kobusingye OC, Hyder AA, Bishai D, et al. Emergency medical systems in low- and middle-income countries: recommendations for action. Bull World Health Organ 2005;83:626-31.

11. Brown AD, Garber AM. Cost effectiveness of coronary heart disease prevention strategies in adults. Pharmacoeconomics 1998;14:27-48.

12. De Smedt D, Kotseva K, De Bacquer D, et al. Cost-effectiveness of optimizing prevention in patients with coronary heart disease: the EUROASPIRE III health economics project. Eur Heart J 2012;33:2865-72.

13. Chola L, Michalow J, Tugendhaft A, et al. Reducing diarrhoea deaths in South Africa: Costs and effects of scaling up essential 
interventions to prevent and treat diarrhoea in under-five children. BMC Public Health 2015;15:394-1689.

14. Vlassoff $C$. Gender differences in determinants and consequences of health and illness. J Health Popul Nutr 2007;25:47-61.

15. Macintyre S, Hunt K, Sweeting $\mathrm{H}$, et al. Gender differences in health: are things really as simple as they seem? Soc Sci Med 1996;42): :617-24.

16. Denton M, Prus S, Walters V. Gender differences in health: a Canadian Study of the psychosocial, structural and behavioural determinants of health. Soc Sci Med 2004;58:2585-600.

17. Salomon JA, Wang $H$, Freeman MK, et al. Healthy life expectancy for 187 countries, 1990-2010: a systematic analysis for the global burden Disease Study 2010. Lancet 2012;380:2144-62.

18. Viner RM, Coffey C, Mathers C, et al. 50-year mortality trends in children and young people: a study of 50 low-income, middleincome, and high-income countries. Lancet 2011;377:1162-74.

19. Wang H, Dwyer-Lindgren L, Lofgren KT, et al. Age-specific and sex-specific mortality in 187 countries, 1970-2010: a systematic analysis for the global burden of Disease Study 2010. Lancet 2012;380:2071-94.

20. GBD 2016 Causes of Death Collaborators. Global, regional, and national age-sex specific mortality for 264 causes of death, 19802016: a systematic analysis for the global burden of Disease Study 2016. Lancet 2017;390:1151-210.

21. Moran AE, Oliver JT, Mirzaie M, et al. Assessing the global burden of ischemic heart disease: Part 1: methods for a systematic review of the global epidemiology of ischemic heart disease in 1990 and 2010. Glob Heart 2012;7:315-29.

22. Forouzanfar $M H$, Moran $A E$, Flaxman $A D$, et al. Assessing the global burden of ischemic heart disease, part 2: Analytic methods and estimates of the global epidemiology of ischemic heart disease in 2010. Glob Heart 2012;7:331-42.

23. Sauser K, Vickery KD, Davis MM. Bottleneck or Magnifying glass? Monitoring the Health-care system's vital signs through emergency departments. Public Health Rep 2015;130:431-4. 